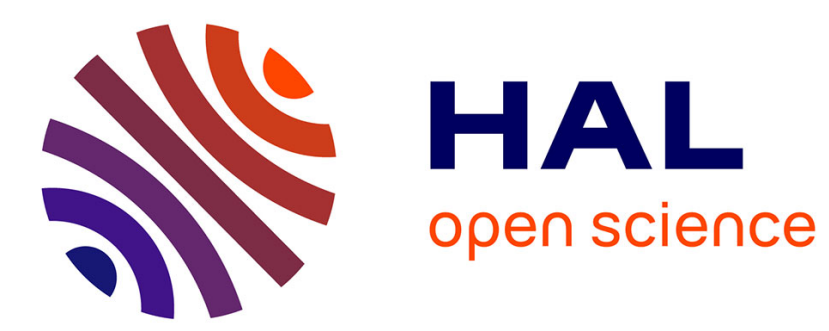

\title{
The Thomas-Fermi theory of atoms and expectation values of electron positions
}

I.K. Dmitrieva, G.I. Plindov, S.K. Pogrebnya

\section{To cite this version:}

I.K. Dmitrieva, G.I. Plindov, S.K. Pogrebnya. The Thomas-Fermi theory of atoms and expectation values of electron positions. Journal de Physique, 1982, 43 (9), pp.1339-1351. 10.1051/jphys:019820043090133900 . jpa-00209513

\section{HAL Id: jpa-00209513 https://hal.science/jpa-00209513}

Submitted on 1 Jan 1982

HAL is a multi-disciplinary open access archive for the deposit and dissemination of scientific research documents, whether they are published or not. The documents may come from teaching and research institutions in France or abroad, or from public or private research centers.
L'archive ouverte pluridisciplinaire HAL, est destinée au dépôt et à la diffusion de documents scientifiques de niveau recherche, publiés ou non, émanant des établissements d'enseignement et de recherche français ou étrangers, des laboratoires publics ou privés. 


\title{
The Thomas-Fermi theory of atoms and expectation values of electron positions
}

\author{
I. K. Dmitrieva $\left({ }^{*}\right)$, G. I. Plindov $\left({ }^{* *}\right)$ and S. K. Pogrebnya $\left({ }^{*}\right)$ \\ (*) A. V. Luikov Heat and Mass Transfer Institute, Byelorussian Academy of Sciences, Minsk 220728, U.S.S.R. \\ $\left.{ }^{* *}\right)$ Nuclear Power Engineering Institute, Byelorussian Academy of Sciences, Minsk, U.S.S.R.
}

(Reçu le 22 février 1982, révisé le 4 mai, accepté le 25 mai 1982)

\begin{abstract}
Résumé. - L'expression analytique de $\left\langle r^{a}\right\rangle$ pour des atomes dans un degré d'ionisation arbitraire est obtenue dans le cadre de la théorie de Thomas-Fermi, compte tenu des corrections dues à l'échange et à l'inhomogénéité de la densité électronique. La contribution des électrons fortement liés à la valeur moyenne $\left\langle r^{a}\right\rangle$ est obtenue lorsque $-5 / 2\left\langle a\left\langle-1 / 2\right.\right.$. La dépendance des coefficients du développement de $\left\langle r^{a}\right\rangle$ en $Z^{-1}$ est donnée explicitement en fonction du nombre d'électrons.
\end{abstract}

\begin{abstract}
Analytical expressions of $\left\langle r^{a}\right\rangle$ for an atom. with arbitrary ionization degree are found within the Thomas-Fermi model including the corrections for exchange and inhomogeneity of the electron density. The contribution of strongly bound electrons is obtained for the expectation values of $r^{a}$ with $-5 / 2<a<-1 / 2$. The $Z^{-1}$ expansion coefficients of $\left\langle r^{a}\right\rangle$ as a function of the electron number are given in an explicit form.
\end{abstract}

1. Introduction. - In spite of the fact that in recent years considerable success has been achieved in the description of the atomic structure within the Hartree-Fock method, interest to the studies of atomic properties with easier methods is still increasing. Especially attractive in this respect is the Thomas-Fermi (TF) model of an atom [1], which provides analytical relations of the physical properties of atomic systems with natural parameters, namely : the electron number $N$ and the nuclear charge $Z$.

The goal of this work is to obtain accurate analytical estimates of the expectation values of $r^{a}$ within the statistical theory including the strongly bound electron contribution.

Consider a system of $N$ electrons at distances $r_{i}(1 \leqslant i \leqslant N)$ from the nucleus. The expectation value of the position operator $r^{a}$ is defined by

$$
\left\langle r^{a}\right\rangle=\sum_{i=1}^{N} \int\left|\Phi\left(r_{1}, \ldots, r_{N}\right)\right|^{2} r_{i}^{a} \mathrm{~d}^{3} r_{1} \ldots \mathrm{d}^{3} r_{N}
$$

where $\Phi\left(r_{1} \ldots r_{i} \ldots r_{N}\right)$ is the wave function of the system. The range of $a$ depends on the wave function behaviour at zero and at infinity and is given by the expression

$$
-3<a<\infty
$$

The main part of $\left\langle r^{a}\right\rangle$ for some a may be obtained within the simple statistical TF model following below.

2. Simple Thomas-Fermi model. - The TF model of atomic systems is based on the assumption that the electron charge in an atom is distributed continuously with the density $\rho(r)$.

In the TF model $\rho(r)$ is related to the dimensionless atomic potential $\Psi(x)[1]$

$$
\rho(r)=\frac{32 Z^{2}}{9 \pi^{3}}\left[\frac{\Psi(x)}{x}\right]^{3 / 2}=\frac{32 Z^{2}}{9 \pi^{3}} \Psi^{\prime \prime}(x) / x .
$$


Here $x$ is the dimensionless radius defined by the expression :

$$
x=\left(9 \pi^{2}\right)^{-1 / 3}(128 Z)^{1 / 3} r=\mu^{-1} r .
$$

$\Psi(x)$ is the solution to the dimensionless TF equation

$$
\Psi^{\prime \prime}(x)=\Psi^{3 / 2}(x) x^{-1 / 2}
$$

with the boundary conditions

$$
\Psi(0)=1, \quad \Psi\left(x_{0}\right)=0, \quad x_{0}^{\prime} \Psi^{\prime}\left(x_{0}\right)=-1+\frac{N}{Z}
$$

where $x_{0}$ is the ion boundary. For a neutral atom $x_{0} \rightarrow \infty$. Here and below atomic units are used.

Using (3) it is easy to obtain the expression for the expectation value of the operator $r^{a}$ in the TF-model :

$$
\begin{aligned}
\left\langle r^{a}\right\rangle_{\mathrm{TF}} & =\int r^{a} \rho(r) \mathrm{d}^{3} r \\
& =\left(9 \pi^{2}\right)^{a / 3}(128)^{-a / 3} Z^{\frac{3-a}{3}} \int_{0}^{x_{0}} \Psi^{3 / 2}(x) x^{a+1 / 2} \mathrm{~d} x .
\end{aligned}
$$

From equations (4) and (5) one may see that both potential $\Psi(x)$ and $x_{0}$ depend on the parameter $N / Z$ alone.

As Lieb and Simon [2] have shown, the TF-model is asymptotically exact in the limit $N \rightarrow \infty$. Therefore, equation (6) gives asymptotically exact estimate of (1) in the range of $a$ where the integral in the right-hand side exists. For a neutral atom, equation (6) immediately gives the asymptotic dependence of $\left\langle r^{a}\right\rangle$ on $Z$

$$
\left\langle r^{a}\right\rangle_{\mathrm{TF}}=A(a) Z^{\frac{3-a}{3}}
$$

where $A(a)$ is calculated numerically using the universal function $\Psi(x)[3]$ :

$$
\begin{aligned}
A(a) & =(3 \pi)^{2 a / 3} 2^{-7 a / 3} \int_{0}^{\infty} \Psi^{3 / 2}(x) x^{a+1 / 2} \mathrm{~d} x \\
& =-(3 \pi)^{2 a / 3} 2^{-7 a / 3} \cdot \frac{3}{2 a+3} \int_{0}^{\infty} \Psi^{1 / 2}(x) \Psi^{\prime}(x) x^{a+\frac{3}{2}} \mathrm{~d} x .
\end{aligned}
$$

Incorrect description of the electron density near the nucleus $\left(\rho(r \rightarrow 0) \sim r^{-3 / 2}\right)$ and at the periphery of a neutral atom $\left(\rho(r \rightarrow \infty) \sim r^{-6}\right)$ in the TF-model restricts the validity of expression (6) to the following range :

$$
-3 / 2<a<3 .
$$

This range includes four moments of the electron density $a=-1,0,1,2$. The zero moment is the normalization integral, $\left\langle r^{2}\right\rangle$ and $\left\langle r^{-1}\right\rangle$ give the diamagnetic susceptibility and the nuclear magnetic shielding constant. The first estimates of these moments were reported as early as in $[1,4]$. More exact recent values of $A(a)$ at $a=-1,1,2$ are presented in table I. The analysis of the Hartree-Fock data has shown that the asymptotic relation $\left\langle r^{2}\right\rangle Z^{-1 / 3}=43.240$ may be obtained at $Z$ far beyond Periodic Table whereas the asymptotic

$$
\left\langle r^{-1}\right\rangle=1.794 Z^{4 / 3}
$$

describes the Hartree-Fock values for $\mathrm{Ne}$ and Rn within $25 \%$ and $10 \%$, respectively.

Now we return to expression (6) for an atom with arbitrary $N / Z$. Since the positive ion radius in the TFmodel is finite, the range of validity of equation (6) is extended to :

$$
-3 / 2<a<\infty .
$$

Table I. - Values $A(a)$ and $A_{q}(a)$.

\begin{tabular}{lcccc}
\hline$a$ & -2 & -1 & 1 & 2 \\
$A(a)$ & -6.03077 & 1.79374 & 3.18734 & 43.2403 \\
$A_{q}(a)$ & $\frac{2}{3} \pi^{2}$ & -1 & - & - \\
\hline
\end{tabular}


Therefore, it is possible to estimate all electron density moments for an ion but $\left\langle r^{-2}\right\rangle$ within the TF-model. It has been shown in [5] that the solution of the TF-equation for an ion may be expressed as :

$$
\Psi(x)=\left(1-\frac{N}{Z}\right) \sum_{k=0}^{\infty} \lambda^{k} \varphi_{k}\left(\frac{x}{x_{0}}\right)
$$

where $\lambda=(1-N / Z)^{1 / 2} x_{0}^{3 / 2}$. The functions $\varphi_{k}\left(x / x_{0}\right)$ are determined from the hierarchy of the differential equations in a closed form (see Appendix).

Using the boundary condition at $x=0$

$$
\left(1-\frac{N}{Z}\right) \sum_{k=0}^{M} \lambda^{k} \varphi_{k}(0) \simeq 1
$$

we obtained the $N / Z$ series expansion of $\lambda$ (consequently, of $x_{0}$ ) at $N / Z<1$. Here we give some of the first terms of $x_{0}(N / Z)$

$$
x_{0}\left(\frac{N}{Z}\right)=\left(\frac{16 N}{\pi Z}\right)^{2 / 3}\left\{1+\left(3-\frac{1024}{45 \pi^{2}}\right) \frac{N}{Z}+\left(\frac{22}{3}-\frac{19019}{90 \pi^{2}}+\frac{2883584}{2025 \pi^{4}}\right) \frac{N^{2}}{Z^{2}}+\cdots\right\} .
$$

Combining equations (6), (8) and (9) yields the expansion of $\left\langle r^{a}\right\rangle_{\mathrm{TF}}$ at small $N / Z$ for arbitrary $a(-3 / 2<a<\infty)$ :

$$
\begin{aligned}
\left\langle r^{a}\right\rangle_{\mathrm{TF}}= & Z^{-a} N^{\frac{2}{3} a+1} \sum_{k=0}^{\infty} A_{k}(a)\left(\frac{N}{Z}\right)^{k}, \\
A_{0}(a)= & \frac{12}{\sqrt{\pi}}(18)^{a / 3} \frac{\Gamma\left(a+\frac{3}{2}\right)}{\Gamma(a+4)}, \\
\frac{A_{1}(a)}{A_{0}(a)}= & \frac{a}{2}+\frac{20 a+24}{\pi^{2}} \sum_{m=0}^{a+1} \frac{(2 m+2) ! !}{(m+3)(2 m+3) ! !}- \\
& \quad-\frac{(2 a+3) 512}{45 \pi^{2}}+\frac{2^{a+5}(a+2) !\left(15 a^{3}+143 a^{2}+398 a+360\right)}{3 \pi^{2}(2 a+5) ! !(a+4)(a+5)} .
\end{aligned}
$$

The expression of $A_{0}(a)$ for arbitrary $a$ was obtained in [6], and the derivation of $A_{1}(a)$ for the integer $a$ is given in Appendix. An equation similar to (11) can be obtained for $A_{2}(a)$ as well. Being bulky, it is omitted here, and we present only numerical estimates of $A_{2}(a)$ alongside $A_{0}(a)$ and $A_{1}(a)$ for $a=-1,1,2,3,4,5,6$ (Table II). It may be seen from table II that for $a \geqslant 2$, the $A_{k}(a)$ grows rapidly with a which restricts the validity of (10) by small $N / Z$.

To improve the estimate (10) for ions with $N / Z \sim 1$, we examine $\left\langle r^{a}\right\rangle_{\mathrm{TF}}$ for a weakly ionized atom.

We express $\Psi(x)$ with $N / Z \sim 1$ as :

$$
\Psi(x) \simeq \Psi_{0}(x)+\Psi_{1}(x)
$$

where $\Psi_{0}(x)$ is a solution of the TF-equation for a neutral atom and $\Psi_{1}(x)$ is a correction function being the solution of the equation obtained in [7]

$$
\Psi_{1}^{\prime \prime}(x)=\frac{3}{2} \Psi_{0}^{1 / 2}(x) x^{-1 / 2} \Psi_{1}(x) .
$$

Table II. - Values $A_{k}(a), A_{k}^{\mathrm{ex}}(a), A_{k}^{\mathrm{KP}}(a)$.

\begin{tabular}{lcccccccc}
\hline$a$ & -2 & -1 & 1 & 2 & \multicolumn{1}{c}{3} & \multicolumn{1}{c}{4} & \multicolumn{1}{c}{5} & \multicolumn{1}{c}{6} \\
\hline$A_{0}$ & -3.49432 & 2.28943 & 0.982777 & 1.28780 & 1.96875 & 3.31687 & 5.97621 & 11.3115 \\
$A_{1}$ & -3.05165 & -0.484895 & 0.491389 & 1.41505 & 3.42557 & 7.96728 & 18.3800 & 42.5968 \\
$A_{2}$ & 0.400220 & 0 & 0.288609 & 1.29718 & 4.29532 & 12.7092 & 35.6340 & 97.0155 \\
$A_{0}^{\text {ex }}$ & & 0.354049 & -0.303964 & -0.876270 & -2.13990 & -5.03168 & -11.7384 & -27.4256 \\
$A_{1}^{\text {ex }}$ & & 0 & -0.328277 & -1.42736 & -4.89936 & -14.9730 & -43.0861 & -119.808 \\
$A_{0}^{\text {KP }}$ & & 0.432726 & -0.343878 & -1.01964 & -2.51630 & -5.95372 & -13.9478 & -32.6864 \\
$A_{1}^{\mathrm{KP}}$ & & 0 & -0.371264 & -1.65477 & -5.73930 & -17.6734 & -51.1114 & -142.621 \\
\hline
\end{tabular}


An asymptotic form of $\Psi_{0}(x)$ and $\Psi_{1}(x)$ at $x \gg 1$ is given by :

$$
\begin{aligned}
& \Psi_{0}(x) \simeq 144 x^{-3}\left(1+0\left(x^{-\sigma}\right)\right), \\
& \Psi_{1}(x) \simeq B x^{4+\sigma}\left(1+0\left(x^{-\sigma}\right)\right)
\end{aligned}
$$

where $\sigma=(\sqrt{73}-7) / 2, B=-2.02 \times 10^{-6}(1-N / Z)^{\frac{7+6}{3}}[8]$.

Substitution of (12) into (6) yields :

$$
\left\langle r^{a}\right\rangle_{\mathrm{TF}}=\left(\frac{9 \pi^{2}}{128}\right)^{a / 3} Z^{\frac{3-a}{3}} \int_{0}^{x_{0}}\left[\Psi_{0}^{3 / 2}(x)+\frac{3}{2} \Psi_{0}^{1 / 2}(x) \Psi_{1}(x)\right] x^{a+\frac{1}{2}} \mathrm{~d} x .
$$

For $-3 / 2<a<3$, expression (14) may be given by :

$$
\begin{aligned}
\left\langle r^{a}\right\rangle_{\mathrm{TF}} \simeq\left(\frac{9 \pi^{2}}{128}\right)^{a / 3} Z^{\frac{3-a}{3}}\left\{\int_{0}^{\infty} \Psi_{0}^{3 / 2}(x) x^{a+1 / 2} \mathrm{~d} x-\right. & \\
& \left.-\int_{x_{0}}^{\infty} \Psi_{0}^{3 / 2}(x) x^{a+1 / 2} \mathrm{~d} x+\frac{3}{2} \int_{0}^{x_{0}} \Psi_{0}^{1 / 2}(x) \Psi_{1}(x) x^{a+\frac{1}{2}} \mathrm{~d} x\right\} .
\end{aligned}
$$

The main contribution to the second and third integrals in (15) gives the range of $x$ near $x_{0}$. Allowing for only the first terms in expansions (13) and the relation $x_{0} \simeq 10.2(1-N / Z)^{-1 / 3}$ for weakly ionized atom [8], we obtain the asymptotic estimate of these integrals :

$$
\begin{aligned}
\left(\frac{9 \pi^{2}}{128}\right)^{a / 3} Z^{\frac{3-a}{3}}\left[-\int_{x_{0}}^{\infty} \Psi_{0}^{3 / 2}(x) x^{a+1 / 2}+\frac{3}{2} \int_{0}^{x_{0}} \Psi_{0}^{1 / 2}(x)\right. & \left.\Psi_{1}(x) x^{a+1 / 2} \mathrm{~d} x\right] \simeq \\
& \simeq\left(\frac{9 \pi^{2}}{128}\right)^{a / 3} Z^{\frac{3-a}{3}}\left[\frac{12^{3}}{a-3} x_{0}^{a-3}+\frac{18 B}{4+\sigma+a} x_{0}^{4+\sigma+a}\right] \\
& \simeq C(a)(Z-N)^{\frac{3-a}{3}} .
\end{aligned}
$$

Equation (16) is based on the first-order correction function and gives only the approximate estimate of $C(a)$. To find accurate values of $C(a)$, an infinite number of correction functions should be considered. Thus, according to (15) and (16)

$$
\left\langle r^{a}\right\rangle_{\mathrm{TF}} \simeq A(a) Z^{\frac{3-a}{3}}+C(a)(Z-N)^{\frac{3-a}{3}}+\cdots .
$$

At $a>3$, the substitution of $x_{0}(N / Z)$ and asymptotic (13) into (14) gives :

$$
\left\langle r^{a}\right\rangle_{\mathrm{TF}} \simeq C(a)(Z-N)^{\frac{3-a}{3}}+\cdots
$$

at $a=3$

$$
\left\langle r^{3}\right\rangle_{\mathrm{TF}} \simeq-\frac{81 \pi^{2}}{2} \ln \left(1-\frac{N}{Z}\right)+C(3)+\cdots .
$$

Expressions (17) and (18) determine approximately the singularity type at $N / Z \rightarrow 1$. It seems therefore useful to express $\left\langle r^{a}\right\rangle_{\mathrm{TF}}$ at $a>3$ as $(N / Z<1)$ :

$$
\left\langle r^{a}\right\rangle_{\mathrm{TF}} \simeq\left(\frac{N}{Z}\right)^{\frac{2 a+3}{3}}(Z-N)^{\frac{3-a}{3}} f\left(\frac{N}{Z}\right)
$$

where $f(N / Z)$ is a function without singularities at $N / Z=0$ and $N / Z=1$. For $a<3$, the convergence of series (10) can be improved using the Padé-approximants. Equations (10) and (17) through (19) define the expectation value $r^{a}$ as a function of $N$ and $Z$ at a large electron number.

3. Improved TF-models. - Expressions (7) and (10) for expectation values can be noticeably improved with allowance for the quantum corrections due to exchange and inhomogeneity of the electron density within the TF-model.

Numerical values of $\left\langle r^{a}\right\rangle$ with the exchange interaction correction are usually obtained on the basis of the 
Thomas-Fermi-Dirac (TFD) equation [9]. The main difference between the TFD and TF-models is that a neutral TFD atom is limited by $x_{0}<\infty$. Therefore, $\left\langle r^{a}\right\rangle$ may be evaluated at $a>3$.

The analytical dependence of the exchange contribution on $N$ and $Z$ may be conveniently obtained by the first-order equation with respect to the exchange parameter, $\beta=\frac{3}{2}(6 \pi Z)^{-2 / 3}[10]$ :

$$
\begin{aligned}
& \Psi(x)=\Psi_{\mathrm{TF}}(x)+\beta \Psi_{\mathrm{ex}}(x) \\
& \rho(r)=\rho_{\mathrm{TF}}+\beta \rho_{\mathrm{ex}}=\frac{32 Z^{2}}{9 \pi^{3}}\left[\frac{\Psi_{\mathrm{TF}}^{\prime \prime}(x)+\beta \Psi_{\mathrm{ex}}^{\prime \prime}(x)}{x}\right] \\
& \Psi_{\mathrm{ex}}^{\prime \prime}(x)-\frac{3}{2} \Psi_{\mathrm{TF}}^{1 / 2}(x) x^{-1 / 2} \Psi_{\mathrm{ex}}(x)=3 \Psi_{\mathrm{TF}}(x) .
\end{aligned}
$$

Using expression (20) for the electron density, we may re-write (6) as :

$$
\left\langle r^{a}\right\rangle=\int r^{a}\left(\rho_{\mathrm{TF}}+\beta \rho_{\mathrm{ex}}\right) \mathrm{d}^{3} r=\left(\frac{9 \pi^{2}}{128}\right)^{a / 3} Z^{\frac{3-a}{3}} \times\left\{\int_{0}^{x_{\mathrm{ex}}} \Psi_{\mathrm{TF}}^{\prime \prime}(x) x^{a+1} \mathrm{~d} x+\beta \int_{0}^{x_{\mathrm{ex}}} \Psi_{\mathrm{ex}}^{\prime \prime}(x) x^{a+1} \mathrm{~d} x\right\} .
$$

Here $x_{\mathrm{ex}}=x_{0}+\Delta_{1} x$ is the boundary radius with the exchange interaction included.

The analysis of $\left\langle r^{a}\right\rangle$ through (21) for a neutral atom was made earlier [10]. Let us repeat the main conclusions of this work. If $a<3$, then $\left\langle r^{a}\right\rangle$ for a neutral atom can be expressed as the sum of two parts :

$$
\left\langle r^{a}\right\rangle=\left\langle r^{a}\right\rangle_{\mathrm{TF}}+\left\langle r^{a}\right\rangle_{\mathrm{ex}}
$$

where

$$
\left\langle r^{a}\right\rangle_{\mathrm{ex}}=\left(\frac{9 \pi^{2}}{128}\right)^{a / 3} Z^{\frac{3-a}{3}}\left[-\int_{x_{\mathrm{ex}}}^{\infty} \Psi_{\mathrm{TF}}^{3 / 2}(x) x^{a+1 / 2} \mathrm{~d} x+3 \beta \int_{0}^{x_{\mathrm{ex}}}\left(\Psi_{\mathrm{TF}}(x) x^{a+1}+\frac{1}{2} \Psi_{\mathrm{TF}}^{1 / 2}(x) \Psi_{\mathrm{ex}}(x) x^{a+\frac{1}{2}}\right) \mathrm{d} x\right]
$$

$x_{\mathrm{ex}} \sim Z^{1 / 3}$ is the boundary radius of a neutral atom [10]. Allowing for the asymptotic behaviour of $\Psi_{\mathrm{ex}} \simeq-27 / x$, one observes that $\left\langle r^{a}\right\rangle_{\mathrm{ex}}$ with $-3 / 2\langle a\langle 1$ may be expressed by :

$$
\left\langle r^{a}\right\rangle_{\mathrm{ex}}=A_{\mathrm{ex}}(a) Z^{\frac{1-a}{3}}+O\left(Z^{\frac{1-a-\sigma}{3}}\right)
$$

where $A_{\mathrm{ex}}(a)$ is calculated numerically with the universal functions $\Psi_{\mathrm{TF}}(x)$ and $\Psi_{\mathrm{ex}}(x)$ :

$$
A_{\mathrm{ex}}(a)=3^{\frac{2 a+1}{3}} \pi^{\frac{2 a-2}{3}} 2^{-\frac{7 a+5}{3}} \int_{0}^{\infty}\left[3 \Psi_{\mathrm{TF}}(x)+\frac{3}{2}\left(\frac{\Psi_{\mathrm{TF}}(x)}{x}\right)^{1 / 2} \Psi_{\mathrm{ex}}(x)\right] x^{a+1} \mathrm{~d} x
$$

For $a=-1, A_{\text {ex }}(-1)=0.45437$

At $1<a<3$ the qualitative relation

$$
\left\langle r^{a}\right\rangle_{\mathrm{ex}} \simeq A_{\mathrm{ex}}(a)
$$

follows from (23). $A_{\mathrm{ex}}(a)$ can be evaluated only approximately.

At $a>3$, equation (22) does not hold because integrals (6) and (23) are divergent. Returning to(21), we obtain the asymptotic

$$
\left\langle r^{a}\right\rangle \simeq A(a)
$$

For ions, equation (22) is valid with any $a$ in the range $-3 / 2<a<\infty$. The correction function $\Psi_{\mathrm{ex}}(x)$ for an ion is expressed as :

$$
\Psi_{\mathrm{ex}}(x)=\left(1-\frac{N}{Z}\right)^{1 / 2} x_{0}^{1 / 2} \sum_{k=1}^{\infty} \lambda^{k} \chi_{k}\left(\frac{x}{x_{0}}\right)
$$

Some of the first $\chi_{k}(y)$ were found in [6] from the inhomogeneous second-order equation hierarchy.

Using the boundary condition at $x=0$, we have found (see Appendix)

$$
\Delta_{1} x=-1286^{-2 / 3} \pi^{-8 / 3} N Z^{-5 / 3}\left[1+\left(\frac{379}{32}-\frac{512}{5 \pi^{2}}\right) \frac{N}{Z}+\cdots\right]
$$


Substituting (27) into (21) and including (28) show that $\left\langle r^{a}\right\rangle_{\mathrm{ex}}$ may be expressed as :

$$
\left\langle r^{a}\right\rangle_{\mathrm{ex}}=N^{\frac{2 a+4}{3}} Z^{-(a+1)} \sum_{k=0}^{\infty} A_{k}^{\mathrm{ex}}(a)\left(\frac{N}{Z}\right)^{k}
$$

We have found for arbitrary $a(-3 / 2<a<\infty)$ :

$$
A_{0}^{\mathrm{ex}}(a)=\frac{48}{\pi^{2}}(18)^{\frac{a-1}{3}}\left[\frac{3}{(a+2)(a+3)}-\frac{4}{\sqrt{\pi}} \frac{\Gamma(a+5 / 2)}{\Gamma(a+4)}\right] .
$$

The function $A_{1}^{\text {ex }}(a)$ for the integer $a$ has been also obtained analytically (see Appendix); the numerical values of $A_{0}^{\mathrm{ex}}(a)$ and $A_{1}^{\mathrm{ex}}(a)$ for $a=-1,1, \ldots, 6$ are given in table II. As can be seen from table II, the inclusion of the exchange interaction increases the expectation value $\left\langle r^{-1}\right\rangle$ and decreases all other $\left\langle r^{a}\right\rangle$. The exchange interaction effect grows with $a$.

The electron density inhomogeneity may be easily included within the Kompaneets-Pavlovsky (KP) equation [11] :

$$
\begin{gathered}
\Psi(x)=\Psi_{\mathrm{TF}}(x)+\beta \Psi_{\mathrm{KP}}(x), \\
\rho(x)=\frac{32 Z^{2}}{9 \pi^{3}}\left[\frac{\Psi_{\mathrm{TF}}^{\prime \prime}(x)+\beta \Psi_{\mathrm{KP}}^{\prime \prime}(x)}{x}\right] \\
\Psi_{\mathrm{KP}}^{\prime \prime}(x)-\frac{3}{2}\left(\frac{\Psi_{\mathrm{TF}}(x)}{x}\right)^{1 / 2} \Psi_{\mathrm{KP}}(x)=\frac{10}{3} \Psi_{\mathrm{TF}}(x)-\frac{x^{5 / 2}}{12 \Psi_{\mathrm{TF}}^{3 / 2}}\left[\frac{\mathrm{d}}{\mathrm{d} x} \frac{\Psi_{\mathrm{TF}}(x)}{x}\right]^{2} .
\end{gathered}
$$

Here $\Psi_{\mathrm{KP}}(x)$ is the function which includes all first-order corrections with respect to $\beta$, i.e. the effects of the exchange interaction and inhomogeneity of the electron density. Equation (31) has been obtained assuming a weak inhomogeneity and is not valid near the nucleus and at the periphery of an atom.

The expression for $\left\langle r^{a}\right\rangle$ in the KP-model coincides with (21) if $\Psi_{\mathrm{ex}} \rightarrow \Psi_{\mathrm{KP}}$ and $x_{\mathrm{ex}} \rightarrow x_{\mathrm{KP}}$ where $x_{\mathrm{KP}}=x_{0}+\Delta_{2} x$ is the boundary radius in this model. tuting

We have found that the solution of the KP-equation may be expressed by $\Psi_{\mathrm{TF}}(x)$ and $\Psi_{\mathrm{ex}}(x)$. Indeed, substi-

$$
\Psi_{\mathrm{KP}}(x)=\frac{1}{3} x^{1 / 2} \Psi_{\mathrm{TF}}^{1 / 2}(x)+\frac{11}{9} \Psi_{\mathrm{ex}}(x)
$$

into (31) shows that (32) is the particular solution of (31) with the boundary conditions :

$$
\Psi_{\mathrm{KP}}(0)=0, \quad \Psi_{\mathrm{KP}}(\infty)=0 .
$$

Using the asymptotics of $\Psi_{\mathrm{TF}}(x)$ and $\Psi_{\mathrm{ex}}(x)$ gives that the asymptotic behaviour $\Psi_{\mathrm{KP}}(x \rightarrow \infty) \simeq-29 / x$ for a neutral atom only differs from $\Psi_{\mathrm{ex}}(x \rightarrow \infty)$ by a factor. Then, from the boundary condition

$$
\Psi_{\mathrm{TF}}\left(x_{\mathrm{KP}}\right)+\beta \Psi_{\mathrm{KP}}\left(x_{\mathrm{KP}}\right)=0
$$

it follows

$$
x_{\mathrm{KP}}(Z \gg 1) \sim Z^{1 / 3}
$$

and therefore the asymptotic estimate $\left\langle r^{a}\right\rangle$ at $a>3$ (26) holds including the inhomogeneity of the electron density.

As with the TFD model at $-3 / 2<a<3$, we represent $\left\langle r^{a}\right\rangle$ in the form :

$$
\left\langle r^{a}\right\rangle=\left\langle r^{a}\right\rangle_{\mathrm{TF}}+\left\langle r^{a}\right\rangle_{\mathrm{KP}}
$$

where

$$
\begin{aligned}
\left\langle r^{a}\right\rangle_{\mathrm{KP}}=\left(\frac{9 \pi^{2}}{128}\right)^{a / 3} Z^{\frac{3-a}{3}}\left\{-\int_{x_{\mathrm{KP}}}^{\infty} \Psi_{\mathrm{TF}}^{\prime \prime}(x) x^{a+1} \mathrm{~d} x+\right. \\
\left.\quad+\frac{11}{9} \beta \int_{0}^{x_{\mathrm{KP}}} \Psi_{\mathrm{ex}}^{\prime \prime}(x) x^{a+1} \mathrm{~d} x+\frac{\beta}{3} \int_{0}^{x_{\mathrm{KP}}} x^{a+1} \frac{\mathrm{d}^{2}}{\mathrm{~d} x^{2}}\left[x \Psi_{\mathrm{TF}}(x)\right]^{1 / 2} \mathrm{~d} x\right\} .
\end{aligned}
$$


The main contribution to the integrals in the right-hand side of (34) gives the range of $x$ near $x_{\mathrm{KP}}$. Allowing for (33) and the asymptotics of $\Psi_{\mathrm{TF}}(x \rightarrow \infty)$ and $\Psi_{\mathrm{ex}}(x \rightarrow \infty)$, we have obtained that at $1<a<3$, the estimate of $\left\langle r^{a}\right\rangle_{\mathrm{KP}}$ has the form of (25).

The leading part of the correction for the exchange and inhomogeneity includes the non-analytical dependence on $x_{\mathbf{K P}}$ in determining $\langle r\rangle_{\mathbf{K P}}$ :

$$
\begin{aligned}
& \langle r\rangle_{\mathrm{KP}}=\left(\frac{9 \pi^{2}}{128}\right)^{1 / 3} Z^{2 / 3}\left[-\int_{x_{\mathrm{KP}}}^{\infty} \Psi_{\mathrm{TF}}^{\prime \prime}(x) x^{2} \mathrm{~d} x+\right. \\
& \left.\quad+\beta \int_{0}^{x_{\mathrm{KP}}} \Psi_{\mathrm{KP}}^{\prime \prime}(x) x^{2} \mathrm{~d} x\right]=\frac{3}{16}\left[\left(\Psi_{\mathrm{KP}}^{\prime \prime} x^{3}\right) \ln x \int_{0}^{x_{\mathrm{KP}}}-\int_{0}^{x_{\mathrm{KP}}} x^{3} \Psi_{\mathrm{KP}}^{\prime \prime}(x) \ln x \mathrm{~d} x-\Psi_{\mathrm{TF}}^{\prime}\left(x_{\mathrm{KP}}\right) x_{\mathrm{KP}}^{2}\right. \\
& \left.\quad+2 \Psi_{\mathrm{TF}}\left(x_{\mathrm{KP}}\right) x_{\mathrm{KP}}^{2}-2 \int_{x_{\mathrm{KP}}}^{\infty} \Psi_{\mathrm{TF}}(x) \mathrm{d} x\right] \simeq-\frac{87}{8} \ln x_{\mathrm{KP}}+C_{1} .
\end{aligned}
$$

Then, according to (33)

$$
\langle r\rangle_{\mathrm{KP}} \simeq-\frac{29}{8} \ln Z+C_{2} .
$$

At $-3 / 2<a<1$, the second and third integrals play a leading part in (35) and the first one is the small correction. These integrals slightly depend on the upper limit that may be therefore extended to infinity. Then,

$$
\left\langle r^{a}\right\rangle_{\mathrm{KP}}=A_{\mathrm{KP}}(a) Z^{\frac{1-a}{3}}+O\left(Z^{\frac{1-a-\sigma}{3}}\right)
$$

where

$$
A_{\mathrm{KP}}(a)=\left(\frac{9 \pi^{2}}{128}\right)^{a / 3}(6 \pi)^{-2 / 3}\left\{\frac{11}{6} \int_{0}^{\infty} \Psi_{\mathrm{ex}}^{\prime \prime}(x) x^{a+1} \mathrm{~d} x+\frac{1}{2} \oint_{0}^{\infty} x^{a+1} \frac{\mathrm{d}^{2}}{\mathrm{~d} x^{2}}\left[\left(\Psi_{\mathrm{TF}} x\right)^{1 / 2}\right] \mathrm{d} x\right\}
$$

The symbol denotes that the divergent part of the third integral in (35) appearing at $-3 / 2<a<-1 / 2$ is omitted. This divergent part is due to the incorrect description of the electron density near the nucleus and must be substituted by the correct contribution of strongly bound electrons. The same approach will be applied to the ionized atoms. Since at $a=-1$ the second integral in (38) is equal to zero, we have :

$$
A_{\mathrm{KP}}(-1)=\frac{11}{9} A_{\mathrm{ex}}(-1)=0.44983 \text {. }
$$

Now consider $\left\langle r^{a}\right\rangle$ for the ions. In this case expression (34) is valid at all $a>-3 / 2$. Using expressions (8), (27) and equation (32), it is easy to obtain $\Psi_{\mathrm{KP}}(x)$ for the ions :

$$
\Psi_{\mathrm{KP}}(x)=\left(1-\frac{N}{Z}\right)^{1 / 2} x_{\mathrm{KP}}^{2}\left\{\frac{1}{3}\left[\sum_{k=0}^{\infty} \lambda^{k} \varphi_{k}\left(\frac{x}{x_{\mathrm{KP}}}\right)\right]^{1 / 2}+\frac{11}{9} \sum_{k=1}^{\infty} \lambda^{k} \chi_{k}\left(\frac{x}{x_{\mathrm{KP}}}\right)\right\} .
$$

Substituting (40) into the boundary condition at $x=0$

$$
\Psi_{\mathrm{TF}}(0)+\beta \Psi_{\mathrm{KP}}(0)=1
$$

yields

$$
\Delta_{2} x=\frac{11}{9} \Delta_{1} x
$$

likewise $\Delta_{1} x$ was derived. Combining expressions (40), (41), (28) and (21) we obtain that the contribution due to the exchange interaction and inhomogeneity of the density for $N$ interacting electrons may be expressed as :

$$
\left\langle r^{a}\right\rangle_{\mathrm{KP}}=N^{\frac{2 a+4}{3}} Z^{-a-1} \sum_{k=0}^{\infty} A_{k}^{\mathrm{KP}}(a)\left(\frac{N}{Z}\right)^{k} .
$$

Expression (42) is of the same form as (29) and the improvement concerns only the coefficient values. We have obtained the analytical expression of $A_{0}^{\mathrm{KP}}(a)$ for integer $a$ which is however omitted to save space and only the numerical values of $A_{0}^{\mathrm{KP}}(a)$ and $A_{1}^{\mathrm{KP}}(a)$ (Table II) are given here. 
Comparison of $A_{k}^{\mathrm{KP}}(a)$ with $A_{k}^{\mathrm{ex}}(a)$ easily shows that the electron density inhomogeneity correction is a small part of the exchange one (below 2/9) for all considered values of $a$.

For $a=-1$

$$
A_{k}^{\mathrm{KP}}(-1)=\frac{11}{9} A_{k}^{\mathrm{ex}}(-1)
$$

at any $k$.

Equation (42) gives the correct estimate of $\left\langle r^{a}\right\rangle_{\mathbf{K P}}$ only for $N$ interacting electrons. The exact result for non-interacting electrons can be easily obtained from the summation over states within quantum theory.

4. Strongly bound electrons. - The estimate of $\left\langle r^{a}\right\rangle$ at $-3<a<-3 / 2$ and the essential improvement of $\left\langle r^{a}\right\rangle$ at $-1 / 2<a<-3 / 2$ can be obtained with the quantum effects near the nucleus taken into account. A correct treatment of the strongly bound electron contribution has been recently suggested by Schwinger [12]. Following Schwinger, we delete from semi-classical integral (6), the incorrectly described contribution of strongly bound electrons with the energy below $(\varepsilon)$. With the electron interaction neglected, it is possible to calculate their contribution by summation over the states of $\left\langle r^{a}\right\rangle_{n l}$ for a hydrogen-like ion :

$$
\left\langle r^{a}\right\rangle_{\mathrm{Sb}}=Z^{-a} \sum_{n=1}^{\left[n^{\prime}\right]} \sum_{l=0}^{n-1}\left\langle r^{a}\right\rangle_{n l} .2(2 l+1)
$$

where $n^{\prime}$ is the maximum value of the main quantum number of strongly bound electrons $n^{\prime}=\left(Z^{2} /-2 \varepsilon\right)^{1 / 2}$; $n^{\prime}$ may be a non-integer; the symbol [ ] denotes an integer part of the number.

The contribution of the bulk electrons is calculated using the TF-model. It is natural that the contribution of strongly bound electrons, once taken into account by equation (44), must be deleted from the TF-contribution. Assuming that they are located at $0 \leqslant x \leqslant x_{m}$ where $x_{m}=2 n^{\prime 2}(Z \mu)^{-1}=-2 Z(\varepsilon \mu)^{-1}$, it is possible to express the TF-contribution as a sum of two parts :

$$
\begin{aligned}
\left\langle r^{a}\right\rangle_{\mathrm{TF}}=\left(\frac{9 \pi^{2}}{128}\right)^{a / 3} Z^{\frac{3-a}{3}}\left\{\int_{x_{m}}^{x_{0}} \Psi_{\mathrm{TF}}^{3 / 2}(x) x^{a+1 / 2} \mathrm{~d} x+\right. & \\
& \left.+\int_{0}^{x_{m}}\left[\left(1+\Psi_{\mathrm{TF}}^{\prime}(0) x\right)^{3 / 2}-\left(1+\frac{\varepsilon \mu x}{2 Z}\right)^{3 / 2}\right] x^{a+1 / 2} \mathrm{~d} x\right\} .
\end{aligned}
$$

In the second integral, the part to be subtracted is a quasi-classical analog of (44); the electrostatic potential $\Psi_{\mathrm{TF}}(x \ll 1)$ is expressed as $\Psi_{\mathrm{TF}}(x)=1+\Psi_{\mathrm{TF}}^{\prime}(0) x$.

The partial integration of the first integral in (45) and the evaluation of the second one up to the terms $Z^{-a-2 / 3}$ give $(a \neq-3 / 2)$ :

$$
\begin{aligned}
\left\langle r^{a}\right\rangle_{\mathrm{TF}}=-\left(\frac{9 \pi^{2}}{128}\right)^{a / 3} \frac{3}{2 a+3} Z^{\frac{3-a}{3}} \int_{0}^{x_{0}} x^{a+3 / 2} \Psi_{\mathrm{TF}}^{1 / 2}(x) \Psi_{\mathrm{TF}}^{\prime}(x) \mathrm{d} x- & -\frac{2^{a+4} \Gamma(a+5 / 2)}{(2 a+3) \sqrt{\pi} \Gamma(a+4)}\left(n^{\prime}\right)^{2 a+3} Z^{-a}
\end{aligned}
$$

The second right-hand term of $(46) \sim\left(n^{\prime}\right)^{2 a+3}$, is cancelled by one part of the strongly bound electron contribution (44). For the integer $a=-1$ and $a=-2$, it can be easily shown by direct summation over $n$ and $l$ using the expressions of $\left\langle r^{a}\right\rangle_{n l}$ from [13] that

$$
\begin{aligned}
\left\langle r^{-2}\right\rangle_{\mathrm{Sb}} & =Z^{2} \sum_{n=1}^{\left[n^{\prime}\right]} \sum_{l=0}^{n-1} \frac{2(2 l+1)}{n^{3}(l+1 / 2)}=Z^{2} \sum_{n=1}^{\left[n^{\prime}\right]} \frac{4}{n^{2}} \\
& \simeq Z^{2}\left(\sum_{n=1}^{\infty} \frac{4}{n^{2}}-\int_{\left[n^{\prime}\right]+1 / 2}^{\infty} \frac{4 \mathrm{~d} n}{n^{2}}\right)=4 Z^{2}\left(\frac{\pi^{2}}{6}-\frac{1}{\left[n^{\prime}\right]+1 / 2}\right) \\
\left\langle r^{-1}\right\rangle_{\mathrm{Sb}} & =Z \sum_{n=1}^{\left[n^{\prime}\right]} \sum_{l=0}^{n-1} \frac{2(2 l+1)}{n^{2}}=2\left[n^{\prime}\right] Z .
\end{aligned}
$$

Approximating the stepwise function $\left[n^{\prime}\right]$ according to [12] by the continuous function $\left[n^{\prime}\right]=n^{\prime}-1 / 2$, one can easily see that throughout $\left\langle r^{a}\right\rangle=\left\langle r^{a}\right\rangle_{\mathrm{Sb}}+\left\langle r^{a}\right\rangle_{\mathrm{TF}}$ the terms depending on $n^{\prime}$ are cancelled out. 
Table III. - Expectation values $\left\langle r^{-1}\right\rangle$ and $\left\langle r^{-2}\right\rangle$ for a neutral atom (Hartree-Fock values are given in brackets [15]).

\begin{tabular}{cccccccc}
\hline$Z$ & 10 & 20 & 30 & 40 & 54 & 86 & 118 \\
\hline$\left\langle r^{-1}\right\rangle 10^{-2}$ & 0.307 & 0.807 & 1.416 & 2.106 & 3.185 & 6.037 & 9.310 \\
& $(0.311)$ & $(0.802)$ & $(1.421)$ & $(2.108)$ & $(3.179)$ & $(6.043)$ & $(9.289)$ \\
$\left\langle r^{-2}\right\rangle 10^{-3}$ & 0.378 & 1.74 & 4.18 & 7.71 & 14.5 & 38.6 & 74.5 \\
& $(0.415)$ & $(1.83)$ & $(4.31)$ & $(7.90)$ & $(14.8)$ & $(39.0)$ & $(75.2)$ \\
\hline
\end{tabular}

In a similar way, for arbitrary $-5 / 2<a<-1 / 2$, we may express $(a \neq-3 / 2)$

$$
\left\langle r^{a}\right\rangle=A_{q}(a) Z^{-a}-\frac{3}{2 a+3}\left(\frac{9 \pi^{2}}{128}\right)^{a / 3} Z^{\frac{3-a}{3}} \int_{0}^{x_{0}} \Psi_{\mathrm{TF}}^{1 / 2}(x) \Psi_{\mathrm{TF}}^{\prime}(x) x^{a+3 / 2} \mathrm{~d} x .
$$

The value $A_{q}(-2)$ obtained in [14] is presented in table I along with $A_{q}(-1)$. Equation (48) includes two terms, namely, quantum and TF ones. At $a>-3 / 2$, the latter coincides with expression (6) obtained previously. The TF-term, in its turn, may be supplemented with the corrections for the exchange and inhomogeneity of the electron density. We will now consider the cases when it is legitimate.

At $-5 / 2<a<-3 / 2$ the quantum contribution $\sim Z^{-a}$ is the main one and $\left\langle r^{a}\right\rangle_{\mathrm{TF}} \sim Z^{(3-a) / 3}$ is a correction for it. The inclusion of $\left\langle r^{a}\right\rangle_{\mathrm{ex}}$ or $\left\langle r^{a}\right\rangle_{\mathbf{K P}}$ is not valid since these terms are of the order of $Z^{(1-a) / 3}$, which is lower than the terms $\sim Z^{-a-(2 / 3)}$ neglected in deriving (48). So, the expectation value $\left\langle r^{a}\right\rangle$ in the range $-3 / 2>a>-5 / 2$ will be determined from :

$$
\left\langle r^{a}\right\rangle=\left\langle r^{a}\right\rangle_{q}+\left\langle r^{a}\right\rangle_{\mathrm{TF}} .
$$

For a neutral atom, $\left\langle r^{a}\right\rangle_{\mathrm{TF}}$ has form (7), and for an ion expression (10) is valid. The values $A(a)$ and $A_{q}(a)$ are presented in tables I and II. In particular, for a neutral atom [14]

$$
\left\langle r^{-2}\right\rangle=\frac{2}{3} \pi^{2} Z^{2}-6.031 Z^{5 / 3} .
$$

In table III the values of $\left\langle\boldsymbol{r}^{-2}\right\rangle$ from (50) are compared with the Hartree-Fock results [15]. It is seen that the error rapidly falls as $Z$ increases, and at $Z \sim 100$ it reaches $1 \%$.

At $-3 / 2\left\langle a<-1 / 2,\left\langle r^{a}\right\rangle_{\mathrm{TF}}\right.$ is the main term, the quantum term is the first correction for it and $\left\langle r^{a}\right\rangle_{\mathrm{KP}}$ is the second correction. In this range of $a$, we can include all three contributions considered in the form :

$$
\left\langle r^{a}\right\rangle=\left\langle r^{a}\right\rangle_{\mathrm{TF}}+\left\langle r^{a}\right\rangle_{q}+\left\langle r^{a}\right\rangle_{\mathrm{KP}}
$$

In particular, combining (48) and (39), we obtain the asymptotic formula for a neutral atom with three exact terms

$$
\left\langle r^{-1}\right\rangle=1.7937 Z^{4 / 3}-Z+0.44983 Z^{2 / 3} .
$$

A comparison with the Hartree-Fock data [15] shows that formula (52) can be used for rapid and reliable estimation of $\left\langle r^{-1}\right\rangle$ beginning with $Z \sim 10$ (Table III).

Finally, at $a\rangle-1 / 2$, the quantum contribution gives a higher-order correction than $\left\langle r^{a}\right\rangle_{\mathrm{KP}}$ and can be omitted. Thus, at $a\rangle-1 / 2,\left\langle r^{a}\right\rangle$ is determined by (34).

Equations (49), (51) and (34) express the expectation values $\left\langle r^{a}\right\rangle$ as functions of $a$ and the ionization degree within the TF-model with the exchange interaction, the inhomogeneity of the electron density and the strongly bound electron contribution included.

5. Relation with $Z^{-1}$ perturbation theory. - As is known [16], $\left\langle r^{a}\right\rangle$ for light atoms may be calculated from $Z^{-1}$ expansion

$$
\left\langle r^{a}\right\rangle=Z^{-a} \sum_{k=0}^{\infty} B_{k}(a, N) Z^{-k}
$$


Exact $B_{0}(a, N)$ can be obtained by summation over the hydrogenlike ion states. The asymptotic function $B_{0}(a, N)$ at $a>-3 / 2$ is given within the TF-model as in [6] :

$$
B_{0}(a, N)=A_{0}(a) N^{\frac{2}{3} a+1}
$$

It has been recently shown [17] that the asymptotic expression of $B_{0}(a, N)$ within the hydrogen-like model fully coincides with (54).

For $a<-1 / 2$, the asymptotic estimate of $B_{0}$ includes the strongly bound electron contribution :

$$
B_{0}(a, N)=A_{0}(a) N^{\frac{2}{3} a+1}+A_{q}(a)
$$

and at $a<-3 / 2$, the second term in (55) becomes the leading one. Accurate calculation of the coefficients $B_{k}(a, N)$ at $k>0$ is very difficult and has been made only for He isoelectronic series. Expressions (10), (29) and (42) give not only the structure but also a reliable estimate of $B_{k}(\mathrm{a}, \mathrm{N})$ :

$$
\begin{aligned}
& B_{k}(a, N)=A_{k}(a) N^{k+\frac{2 a+1}{3}}, \quad-5 / 2<a<-3 / 2 \\
& B_{k}(a, N)=A_{k}(a) N^{k+\frac{2 a+1}{3}}+A_{k-1}^{\mathrm{KP}}(a) N^{k+\frac{2 a+1}{3}} \quad a>-3 / 2 .
\end{aligned}
$$

Comparing the asymptotic $B_{0}(a, N)$ from (54) and (55) with the exact ones, we have found that for $a>0$ there exists an oscillatory part of the relative order $N^{-1 / 3}$ and for $a<0$ its relative order is $N^{-2 / 3}$ (Fig. 1). We propose that the behaviour of the oscillatory contribution to $B_{k}(a, N)$ with $k \geqslant 1$ is similar.

An important property of the $Z^{-1}$ expansion coefficients follows from expression (56) : the

$$
B_{k+1}(a, N) / N B_{k}(a, N)
$$

ratio rapidly tends to the constant given by the TF-model at $N \rightarrow \infty$. In figure 2 the $B_{2} / N B_{1}=f(a, N)$ ratios are given with the corrections for the exchange and inhomogeneity of the electron density. It is seen that for the considered values of a the effects due to quantum corrections are essential for $N \leqslant 10$ only. We expect that within the TF-model the ratios of $Z^{-1}$ expansion coefficients are reproduced better than the coefficients themselves.

The most accurate coefficients in $Z^{-1}$ expansion can be obtained for the expectation values in the range $-3 / 2<a<-1 / 2$ as follows from (51). For example, for $\left\langle r^{-1}\right\rangle$, combining (48), (10) and (42), we obtain :

$$
\left\langle r^{-1}\right\rangle=\left(2.2894 N^{1 / 3}-1\right) Z-\left(0.48489 N^{4 / 3}-0.432726 N^{2 / 3}\right) \text {. }
$$

The comparison of $\left\langle r^{-1}\right\rangle$ from (57) for Ar isoelectronic series with the Hartree-Fock results [15] shows that the quantum mechanical $\left\langle r^{-1}\right\rangle$ are well reproduced by expression (57) over the whole range of $N$ and $Z$ even for unclosed electron shells (Table IV).

Values of $\left\langle r^{a}\right\rangle$ for ions at $-5 / 2<a<-3 / 2$ are described by equation (49). Since in (49) the corrections $Z^{-a-(2 / 3)}$ are not included, its accuracy is lower than that of (57). However, in this case a reliable estimate of $\left\langle r^{a}\right\rangle$ may be also obtained (see Table IV).

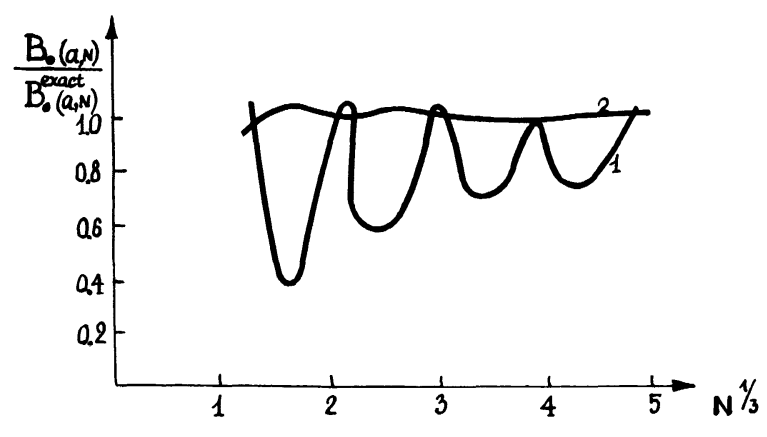

Fig. 1. - The ratios of $B_{0}(a, N)$ from (54)-(55) to exact ones as a function of $N: 1, a=2 ; 2, a=-1$.

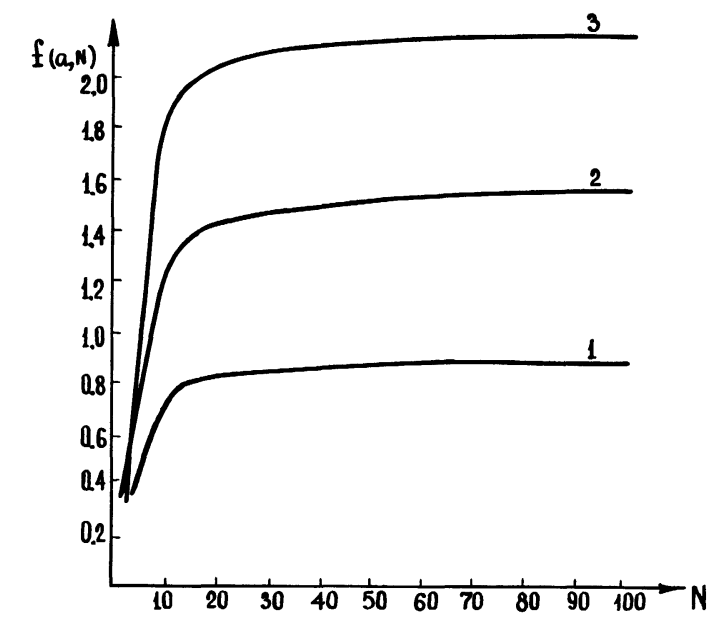

Fig. 2. - The $B_{2} / N B_{1}$ as a function of $N: 1, a=2 ; 2$, $a=4 ; 3, a=6$. 
Table IV. - Expectation values $\left\langle r^{-1}\right\rangle$ and $\left\langle r^{-2}\right\rangle$ for Ar isoelectronic series (Hartree-Fock values are given in brackets [15]).

\begin{tabular}{cccccccc}
\hline$Z$ & 18 & 22 & 26 & 30 & 34 & 38 & $\infty$ \\
\hline$\left\langle r^{-1}\right\rangle Z^{-1}$ & 3.89 & 4.09 & 4.23 & 4.34 & 4.41 & 4.48 & 5.00 \\
& $(3.87)$ & $(4.06)$ & $(4.19)$ & $(4.29)$ & $(4.36)$ & $(4.41)$ & $(4.89)$ \\
$\left\langle r^{-2}\right\rangle Z^{-2}$ & 4.28 & 4.40 & 4.51 & 4.60 & 4.67 & 4.73 & 5.25 \\
& $(4.52)$ & $(4.64)$ & $(4.73)$ & $(4.79)$ & $(4.84)$ & $(4.89)$ & $(5.50)$ \\
\hline
\end{tabular}

For positive exponents the validity of formulas (34), (54) and (56) is limited by two factors. First, because of the small number of terms in $Z^{-1}$ expansion with quickly increasing coefficients these expressions can be used only for multicharged ion properties. This disadvantage is also inherent in $Z^{-1}$ expansion with exact coefficients already at $N \sim 10$ and can be overcome using expression (19) or Padé-approximants of series (53).

The other disadvantage is peculiar of the TF-model and results from the absence of oscillatory contributions to $B_{k}(a, N)$ in expressions $(54)$ and $(56)$.

6. Conclusion. - To conclude the paper we repeat the main results of this work :

1) the expectation values of $r^{a}$ are studied as a function of $a$ and the ionization degree within the TF-model;

2) the analytical expressions for the corrections due to exchange and inhomogeneity of the electron density are given;

3 ) it is shown that the inclusion of a strongly bound electron contribution provides a reliable estimate of $\left\langle r^{a}\right\rangle$ for negative exponents at $-5 / 2<a<-3 / 2$ and essentially improves it at $-3 / 2<a<-1 / 2$;

4) analytical dependences of the $Z^{-1}$ expansion coefficients of $\left\langle r^{a}\right\rangle$ on $N$ and $a$ are obtained.

Acknowledgments. - The authors are grateful to Dr. L. Bartolotti who has kindly sent them the HartreeFock expectation values.

Appendix. - Substituting (8) into TF-equation (4) and setting the coefficients equal at equal powers of $\lambda$ give a hierarchy of the equations [5] :

$$
\varphi_{0}^{\prime \prime}(y)=0, \quad \varphi_{1}^{\prime \prime}(y)=\varphi_{0}^{3 / 2}(y) y^{-1 / 2}, \quad \varphi_{2}^{\prime \prime}(y)=\frac{3}{2} \varphi_{0}^{1 / 2}(y) \varphi_{1}(y) y^{-1 / 2} .
$$

We present the first $\varphi_{k}(y)$ :

$$
\begin{aligned}
\varphi_{0} & =1-y \\
\varphi_{1} & =\left(\frac{1}{8}-\frac{3}{4} y\right) \arccos \sqrt{y}+\frac{1}{24} y^{1 / 2}\left(3+16 y-4 y^{2}\right)(1-y)^{1 / 2} .
\end{aligned}
$$

The subsequent $\varphi_{k}(y)$ values have the same structure. Further, we will need

$$
\varphi_{2}(0)=\frac{2}{15}-\frac{3 \pi^{2}}{256} \quad \text { and } \quad \varphi_{3}(0)=\frac{13 \pi}{16}\left(\frac{\pi^{2}}{256}-\frac{39}{1024}\right) .
$$

From the condition $\Psi(0) \simeq\left(1-\frac{N}{Z}\right) \sum_{k=0}^{3} \lambda^{k} \varphi_{k}(0) \simeq 1$ we get the expansion

$$
\lambda=\frac{16}{\pi} \frac{N}{Z}\left[1+\left(4-\frac{512}{15 \pi^{2}}\right) \frac{N}{Z}+\left(12-\frac{21067}{60 \pi^{2}}+\frac{524288}{225 \pi^{4}}\right) \frac{N^{2}}{Z^{2}}+\cdots\right] .
$$

Substituting (8) into (6) and taking (A .2) and (9) into account, we obtain :

$$
\begin{aligned}
\left\langle r^{a}\right\rangle_{\mathrm{TF}}=\frac{16}{\pi}(18)^{a / 3} N^{\frac{2 a}{3}+1} Z^{-a}\{1+ & \left.\frac{2 a+3}{2}\left(3-\frac{1024}{45 \pi^{2}}\right) \frac{N}{Z}+\cdots\right\} \times \\
\times & {\left[1-\frac{3}{2} \frac{N}{Z}+\cdots\right]\left[J_{0}(a)+\frac{3}{2} \lambda J_{1}(a)+\frac{3}{2} \lambda^{2} J_{2}(a)+\cdots\right] }
\end{aligned}
$$




$$
\begin{aligned}
& J_{0}(a)=\int_{0}^{1} \varphi_{0}^{3 / 2}(y) y^{a+1 / 2} \mathrm{~d} y, \\
& J_{1}(a)=\int_{0}^{1} \varphi_{0}^{1 / 2}(y) \varphi_{1}(y) y^{a+1 / 2} \mathrm{~d} y, \\
& J_{2}(a)=\int_{0}^{1}\left[\varphi_{0}^{1 / 2}(y) \varphi_{2}(y)+\frac{1}{4} \varphi_{1}^{2}(y) \varphi_{0}^{-1 / 2}(y)\right] y^{a+1 / 2} \mathrm{~d} y .
\end{aligned}
$$

Equation (A.3) can be expressed :

$$
\left\langle r^{a}\right\rangle_{\mathrm{TF}}=\frac{16}{\pi}(18)^{a / 3} \frac{\Gamma(5 / 2) \Gamma(a+3 / 2)}{\Gamma(a+4)} N^{\frac{2}{3} a+1} Z^{-a}\left(1+\frac{A_{1}(a)}{A_{0}(a)} \frac{N}{Z}+\frac{A_{2}(a)}{A_{0}(a)} \frac{N^{2}}{Z^{2}}+\cdots\right)
$$

where

$$
\frac{A_{1}(a)}{A_{0}(a)}=3 a+3-\frac{(2 a+3) 512}{45 \pi^{2}}+\frac{24}{\pi} \frac{J_{1}(a)}{J_{0}(a)} .
$$

$J_{0}(a)=\Gamma(5 / 2) \Gamma(a+3 / 2) \Gamma^{-1}(a+4)$, where $\Gamma(x)$ is the gamma function; $J_{1}(a)$ was calculated using the recurrence relation :

$$
J_{1}(a+2)=\int_{0}^{1}(1-y)^{1 / 2} y^{a+3 / 2} \arccos \sqrt{y} \mathrm{~d} y=\frac{2 a+3}{2 a+6} J_{1}(a+1) .
$$

Upon calculations, we have :

$$
\begin{aligned}
J_{1}(a)=\frac{(5 a+6)(2 a+1) ! !}{4(2 a+6) ! !}\left[\sum_{m=0}^{a+1} \frac{(2 m+2) ! !}{(m+3)(2 m+3) ! !}-\right. & \left.-\frac{\pi^{2}}{4}\right]+ \\
& +\frac{15 a^{3}+143 a^{2}+398 a+360}{24(a+2)(a+3)^{2}(a+4)(a+5)} .
\end{aligned}
$$

The substitution of (A.5) into (A.4) gives equation (11). In particular,

$$
\frac{A_{1}(1)}{A_{0}(1)}=\frac{1}{2} ; \frac{A_{1}(2)}{A_{0}(2)}=1+\frac{512}{525 \pi^{2}} .
$$

The values of $A_{2}(a)$ are calculated from the numerical estimates of $J_{2}(a)$.

In order to obtain the functions $\Delta_{1}(x)(N, Z)$ and $A_{1}^{\text {ex }}(a)$ with the exchange included in the first order, the function $\chi_{1}(y)=\frac{1}{2}(1-y)^{3}$ and $\chi_{2}(0)=\frac{27}{1024}$ are necessary [6].

Using the boundary condition at $x=0$

$$
\Psi_{\mathrm{TF}}(0)+\beta \Psi_{\mathrm{ex}}(0)=1
$$

and bearing in mind that $\lambda \simeq\left(1-\frac{N}{Z}\right)^{1 / 2}\left(x_{0}^{3 / 2}+\frac{3}{2} x_{0}^{1 / 2} \Delta_{1} x\right)$, we find the relation of $\Delta_{1} x$ with $x_{0}$ and $N / Z$ : $\left[\frac{3}{2}\left(1-\frac{N}{Z}\right)^{1 / 2} x_{0}^{1 / 2} \varphi_{1}(0)+3\left(1-\frac{N}{Z}\right) x_{0}^{2} \varphi_{2}(0)\right] \Delta_{1} x+\beta x_{0}^{2}\left[\chi_{1}(0)+\left(1-\frac{N}{Z}\right)^{1 / 2} x_{0}^{3 / 2} \chi_{2}(0)\right]=0$.

The $N / Z$ series expansion of(A .6) including (9) and setting the coefficients equal at equal powers of $N / Z$ give (28). One can see from (21) that when the exchange is taken into account in the first order with respect to $\beta$, the following additional contribution to $\left\langle r^{a}\right\rangle$ appear :

1) a contribution due to $x_{0} \rightarrow x_{0}+\Delta_{1} x$ and $\lambda \rightarrow \lambda+\Delta \lambda$

$$
\Delta_{1}\left\langle r^{a}\right\rangle=\left(1-\frac{N}{Z}\right)^{3 / 2} x_{0}^{a+1 / 2}\left[\left(a+\frac{3}{2}\right) J_{0}(a) \Delta_{1} x+\frac{9}{2} J_{1}(a) \Delta \lambda+\frac{3}{2} \frac{\lambda}{x_{0}} J_{1}(a) \Delta_{1} x\right]
$$


2) a contribution of $\sim \beta$

$$
\begin{aligned}
\Delta_{2}\left\langle r^{a}\right\rangle=Z^{\frac{1-a}{3}}\left(\frac{3}{32 \pi^{2}}\right)^{1 / 3}\left(\frac{9 \pi^{2}}{128}\right)^{a / 3}\left(1-\frac{N}{Z}\right) x_{0}^{a+2} & {\left[3 \int_{0}^{1} \varphi_{0}(y) y^{a+1} \mathrm{~d} y+\right.} \\
& \left.+\frac{3}{2} \lambda \int_{0}^{1} \sqrt{\frac{\varphi_{0}}{y}} \chi_{1}(y) y^{a+1} \mathrm{~d} y+3 \lambda \int_{0}^{1} \varphi_{1}(y) y^{a+1} \mathrm{~d} y\right] .
\end{aligned}
$$

Upon appropriate calculations, we have :

$$
\left\langle r^{a}\right\rangle_{\mathrm{ex}}=N^{\frac{2}{3} a+4} Z^{-a-1}\left(A_{0}^{\mathrm{ex}}(a)+A_{1}^{\mathrm{ex}}(a) \frac{N}{Z}\right)
$$

where $A_{0}^{\mathrm{ex}}$ is determined from relation (30),

$$
\begin{aligned}
A_{1}^{\mathrm{ex}}(a)=\frac{256}{\pi^{3}}(18)^{\frac{a-1}{3}}\left\{-\frac{\Gamma(5 / 2) \Gamma(a+5 / 2)}{\Gamma(a+5)}\left[\frac{379+96 a}{32}-\frac{5120+1024 a}{45 \pi^{2}}\right]-\right. \\
\quad-\frac{24(a+3)}{\pi} J_{1}(a)+\frac{9}{4} \frac{\Gamma(9 / 2) \Gamma(a+3 / 2)}{\Gamma(a+6)} \\
\left.+\frac{9 \Gamma(9 / 2) \Gamma(a+1 / 2)}{(a+2)(a+3) \Gamma(a+6)}+\frac{9 \pi(3 a+5)}{16(a+2)(a+3)}-\frac{64}{5 \pi(a+3)}\right\}
\end{aligned}
$$

with $J_{1}(a)$ from (A.5). For example, $A_{1}^{\mathrm{ex}}(1)=\frac{339}{32 \pi^{2}}-\frac{2048}{15 \pi^{4}} ; \quad A_{1}^{\mathrm{ex}}(2)=\frac{18^{1 / 3}}{\pi^{2}}\left(\frac{4509}{320}-\frac{100864}{525 \pi^{2}}\right)$.

\section{References}

[1] Gombâ̂, P., Die statistische Theorie des Atoms und ihre Anwendungen (Springer-Verlag, Wien) 1949.

[2] Lieb, E. H. and Simon, B., Adv. Math. 23 (1977) 22.

[3] Kobayashi, S., Matsukuma, T., Nagai, S. and UMEDA, K., J. Phys. Soc. Japan 10 (1955) 759.

[4] Scott, J. M. C., Phil. Mag. 43 (1952) 853.

[5] Plindov, G. I. and Dmitrieva, I. K., Dokl. Akad. Nauk BSSR 19 (1975) 788.

[6] Dmitrieva, I. K., Plindov, G. I. and Chevganov, B. A., Opt. Spectrosk. 42 (1977) 7.

[7] Fermi, E., Mem. Acc. Italia 1 (1930) 1.

[8] Plindov, G. I. and Dmitrieva, I. K., Dokl. Akad. Nauk BSSR 21 (1977) 209.

[9] Thomas, L. H., J. Chem. Phys. 22 (1954) 1758.

Umeda, K. and Thomas, L. H., J. Chem. Phys. 24 (1956) 1113.
[10] Dmitrieva, I. K. and Plindov, G. I., Izy. Akad. Nauk SSSR, ser. Fiz. 41 (1977) 2639.

[11] Kompaneets, A. S. and Pavlovsky, B. S., Zh. Teor. Fiz. 31 (1956) 427.

[12] Schwinger, J., Phys. Rev. A 22 (1980) 1827.

[13] Bethe, H. A. and SAlPeTER, E. E., Quantum mechanics of one- and two-electron atoms (Springer-Verlag, Berlin) 1957.

[14] Dmitrieva, I. K. and Plindov, G. I., Z. Phys. (in press).

[15] Bartolotti, L. J. (unpublished).

[16] Dalgarno, A. and Stewart, A. L., Proc. Roy. Soc. A 247 (1960) 245.

[17] Tal, Y. and Bartolotti, L. J., J. Chem. Phys. (in press). 